\title{
EFEITO DO TIPO DE TRATAMENTO TÉRMICO E DE SEUS PARÂMETROS NA DUREZA DOS CONSTITUINTES PRESENTES EM AÇOS BIFÁSICOS*
}

Nayara Aparecida Neres da Silva ${ }^{1}$ Pedro Penna Coelho Lourenço ${ }^{2}$ Wellington Lopes ${ }^{3}$

Carlos Eduardo dos Santos ${ }^{4}$ Elaine Carballo Siqueira Corrêa ${ }^{5}$ Cintia Braga ${ }^{6}$

\section{Resumo}

A busca por redução de peso e maior segurança dos veículos vem impulsionando a obtenção de novos aços de elevado desempenho. Os aços bifásicos vêm sendo desenvolvidos e utilizados devido a sua considerável resistência mecânica e boa conformabilidade. No presente trabalho foram realizados os recozimentos intercríticos direto e contínuo em um aço de composição 0,121\%C-0,499\%Si$1,58 \% \mathrm{Mn}-0,021 \% \mathrm{Cr}$ com o objetivo de avaliar os efeitos do tempo e temperatura de intercrítica na dureza da ferrita e do constituinte MA (martensita e austenita retida). A avaliação dos resultados foi realizada por meio de microscopia óptica, microscopia eletrônica de varredura e ensaios de ultramicrodureza. Foi observada a influência do tempo e da temperatura na morfologia e na quantidade de constituintes formados. Também foram verificados diferentes valores de dureza nos constituintes e ainda em distintas regiões dos grãos de ferrita. Os resultados indicaram maiores durezas para regiões próximas da interface ferrita/martensita e diminuição de dureza do constituinte MA e aumento dos valores na ferrita com a elevação do tempo.

Palavras-chave: Aços bifásicos; Microestrutura; Ultramicrodureza.

\section{EFFECT OF THE TYPE OF HEAT TREATMENT AND THEIR PARAMETERS IN THE HARDNESS OF THE CONSTITUENTS PRESENT IN DUAL PHASE STEELS}

\section{Abstract}

The search for weight reduction and greater safety of vehicles has been driving the acquisition of new high performance steels. Dual phase steels have been developed and used due to their considerable mechanical strength and good formability. In the present work, direct and continuous intercritical annealing were carried out in a $0,121 \% \mathrm{C}-0,499 \% \mathrm{Si}-1,58 \% \mathrm{Mn}-0,021 \% \mathrm{Cr}$ steel in order to evaluate the effects of time and temperature on the on the hardness of the constituents present in dual phase steels; ferrite and MA constituent (martensite and residual austenite). The evaluation was performed through optical and scanning electron microscopy and ultramicrohardeness tests. The influence of time and temperature on the constituents was observed. Distinct values of hardness in the constituents and in different ferrite regions were verified. There were higher hardness results for regions near to the interface ferrite/martensite. A decrease of hardness in the MA constituent and an increase in the ferrite with the increase of the holding time was observed.

Keywords: Dual Phase steels; Microstructure; Ultramicrohardeness.

Eng. Metalurgista, Mestre, CEFET-MG, Belo Horizonte, MG, Brasil.

Graduando Eng. de Materiais, CEFET-MG, Belo Horizonte, MG.

Eng. Mecânico, Dr, Professor, Depto Engenharia Materiais, CEFET-MG, Belo Horizonte, MG.

Eng. Mecânico, Mestre, Professor, Depto Engenharia Materiais, CEFET-MG, Belo Horizonte, MG.

Eng. Mecânica, Dra., Professora, Depto Engenharia Materiais, CEFET-MG, Belo Horizonte, MG.

Engenharia de Materiais, mestranda, CEFET-MG, Belo Horizonte, MG, Brasil. 


\section{INTRODUÇÃO}

O interesse da indústria automobilística em aços com melhores propriedades mecânicas, baixo custo e menores espessuras, vêm impulsionando cada dia mais o desenvolvimento e estudo dos aços avançados de alta resistência (Advanced High Strenght Steels - AHSS) [1,2]. A melhora dessas propriedades e a redução de peso, que tem como objetivo a redução do consumo de combustíveis e aumento da segurança dos passageiros, dificultam $o$ processo de estampagem e a conformabilidade desse material [3,4]. Uma alternativa para a solução deste problema é o ajuste da composição química e da rota de processamento do aço. Assim, os aços bifásicos, também conhecidos como aços dual phase (DP) vem sendo desenvolvidos em busca desse balanço de consideráveis propriedades mecânicas, boa conformabilidade e baixo custo se comparado a outros AHSS $[5,6]$.

Os aços dual phase apresentam como composição química típica, um baixo teor de carbono, presença de certas quantidades de manganês (entre $1 \%$ e $2 \%$ ) e outros elementos de liga como silício, nióbio, molibdênio e cromo. Com microestrutura típica composta por uma matriz ferrítica com ilhas de martensita presentes ao longo dessa matriz, também chamado de constituinte MA (martensita + austenita) podendo ainda apresentar outros constituintes em menores proporções [7].

Existem basicamente três tipos de processamentos térmicos utilizados para a obtenção de aços dual phase, eles são: (a) o tratamento intercrítico, também chamado de recozimento intercrítico direto, amplamente utilizado para a produção comercial, que consiste basicamente no aquecimento do material até a região intercrítica onde as fases ferrita+ austenita $(\alpha+\gamma)$ se encontram estáveis, seguido de um resfriamento brusco, fornecendo energia suficiente para a transformação da austenita em martensita [8]; (b) recozimento intercrítico contínuo, que consiste no aquecimento do material até a completa austenitização, seguido de um resfriamento até uma temperatura intercrítica, entre as linhas $A_{c 1}$ e $A_{c 3}$ do diagrama ferrocementita, com a presença das fases austenita+ferrita, ou até a região onde as fases ferrita e cementita coexistem (abaixo da linha $A_{c 1}$ ), seguidos com um posterior resfriamento brusco [9] e (c) tratamento termomecânico de aço baixo teor de carbono, muito utilizado na indústria siderúrgica, com a presença de uma etapa de laminação a frio durante o processamento [7].

A microestrutura formada após esse processamento é influenciada por algumas variáveis presentes no processo, como a microestrutura inicial do aço, presença de elementos de liga, tempo e temperatura de processamento e a taxa de resfriamento, formando microestruturas distintas e como consequência alterando as propriedades do aço obtido [10].

Dentro desse contexto, o presente trabalho tem como objetivo estudar a influência do tipo de tratamento, do tempo encharque e da temperatura de processamento, na microestrutura formada e na dureza de cada um dos constituintes, por meio de ensaios de ultramicrodureza (penetração instrumentada).

\section{MATERIAIS E MÉTODOS}

\subsection{Materiais}

Para a realização dos experimentos, foram adquiridas chapas de aços originalmente bifásicos com $2 \mathrm{~mm}$ de espessura com a composição química (\% em peso) mostrada a Tabela 1. 
Tabela 1: Porcentagem em peso dos elementos químicos no aço inicial

\begin{tabular}{cccccccc}
\hline Elemento & $\mathbf{C}$ & $\mathbf{S i}$ & $\mathbf{M n}$ & $\mathbf{P}$ & $\mathbf{M o}$ & $\mathbf{C r}$ & $\mathbf{N i}$ \\
\hline \% em peso & 0,121 & 0,499 & 1,58 & 0,0108 & 0,001 & 0,0207 & 0,0306 \\
\hline Elemento & $\mathbf{V}$ & $\mathbf{T i}$ & $\mathbf{B}$ & $\mathbf{A l}$ & $\mathbf{N b}$ & $\mathbf{C u}$ & $\mathbf{W}$ \\
\hline$\%$ em peso & 0,0065 & 0,0022 & $<0,0004$ & 0,0529 & 0,0138 & 0,009 & 0,01 \\
\hline
\end{tabular}

\subsection{Tratamentos Térmicos}

As chapas de aço foram submetidas a um processo de normalização a $880^{\circ} \mathrm{C}$ por 60 min, com o intuito de apagar os efeitos do processamento anterior, seguido de dois ciclos de processamentos térmicos distintos, recozimento intercrítico direto e recozimento intercrítico contínuo, ambos em duas temperaturas distintas, $740^{\circ} \mathrm{C}$ e $780^{\circ} \mathrm{C}$, em tempos de encharque de 2 e 30 min. Todos os processos foram realizados em banho de sal, exemplificados nas figuras 1 e 2 .

As temperaturas de processamento foram determinadas a partir das temperaturas de transformação obtidas através do teor de elementos de liga apresentados na tabela 1. Os efeitos desses elementos nas temperaturas de transformação e como foram realizados os cálculos das mesmas são apresentados por Tasca e Machado [11], Gaheri et. al [12] e Movahed et. al. [13], respectivamente.

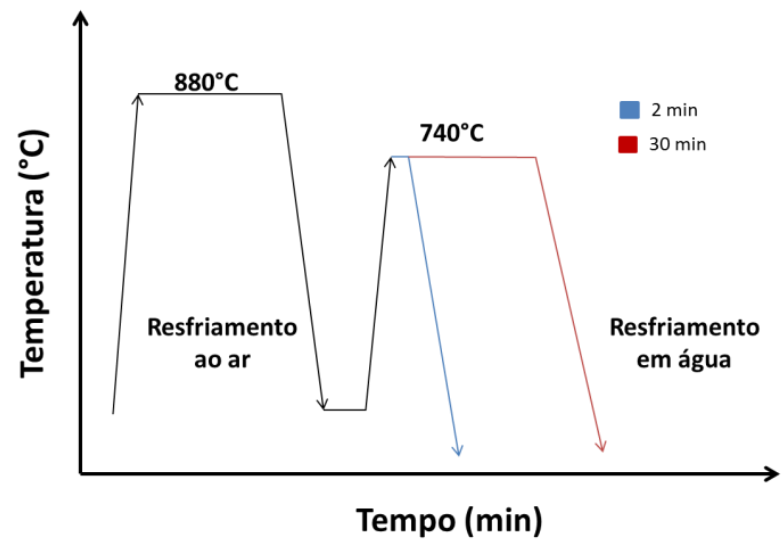

(a)

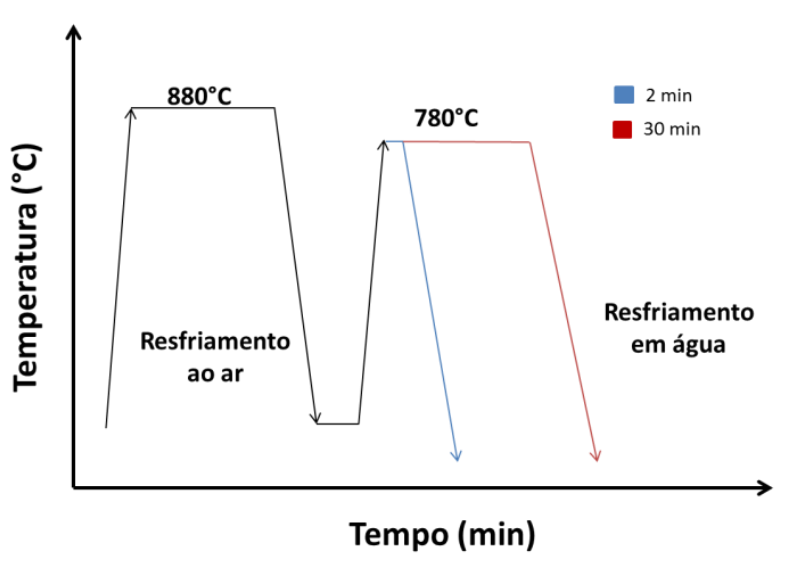

(b)

Figura 1. Processamento Térmico de Recozimento Intercrítico Direto (a) $740^{\circ} \mathrm{C} \mathrm{e} \mathrm{(b)} 780^{\circ} \mathrm{C}$.

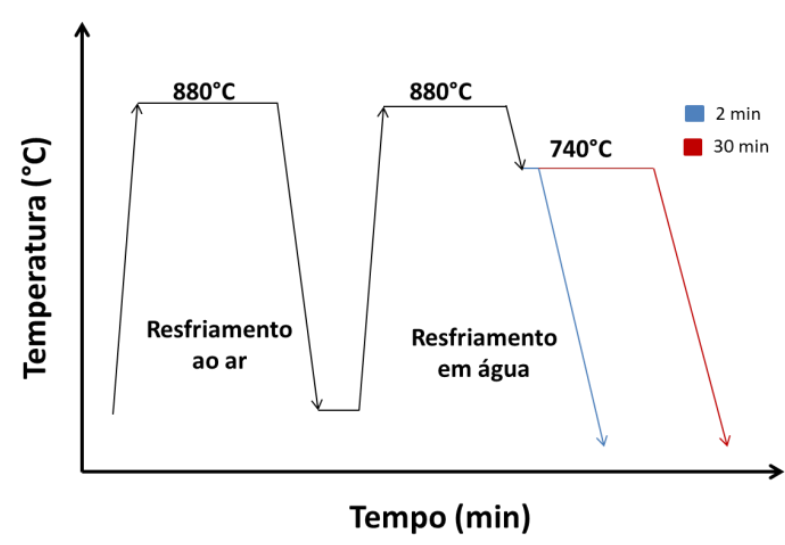

(a)

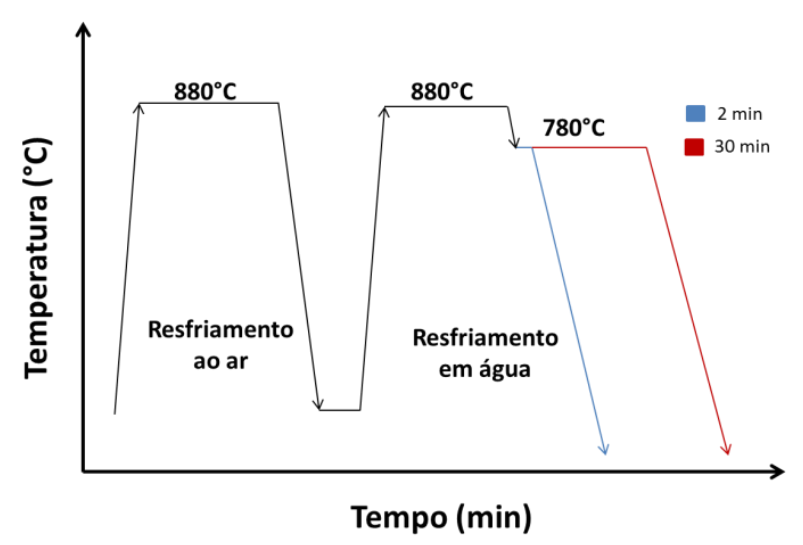

(b)

Figura 2. Processamento Térmico de Recozimento Intercrítico Contínuo (a) $740^{\circ} \mathrm{C} \mathrm{e} \mathrm{(b)} 780^{\circ} \mathrm{C}$. 


\subsection{Caracterização Microestrutural - Fração Volumétrica dos Constituintes}

A caracterização microestrutural das amostras após o processamento térmico foi realizada por meio de procedimentos metalográficos. As amostras foram submetidas a dois ataques químicos distintos, um com o reagente Nital $3 \%$ e o outro com o reagente LePera, com 0 intuito de revelar os constituintes ferrita e MA (martensita+austenita retida) em diferentes colorações [14,15].

Após esses ataques, foi possível analisar de forma qualitativa e semiquantitativa as proporções dos constituintes formados em cada ciclo de processamento, empregando a técnica de contagem por pontos com imagens obtidas no microscópio óptico e no microscópio eletrônico de varredura (MEV). [16]

\subsection{Caracterização Mecânica - Ultramicrodureza (Pentração Instrumentada)}

Com o intuito de avaliar as propriedades dos constituintes separadamente e a influência dos processamentos térmicos em cada um deles, foram realizados ensaios de ultramicrodureza Vickers em diferentes regiões da ferrita e da martensita. As indentações na ferrita foram realizadas nas amostras atacadas com Nital $3 \%$, enquanto as indentações realizadas no constituinte MA foram realizadas nas amostras atacadas com o reagente LePera.

Para todas as amostras uma carga de $30 \mathrm{gf}$ foi aplicada, sendo $5 \mathrm{~s}$ o tempo de carga e 5 s o tempo de descarga. Foram realizadas seis indentações para todas as regiões analisadas na amostra: centro da ferrita, região da ferrita próxima à interface e o constituinte MA. Foram realizados posteriormente os cálculos dos valores médios de dureza e seus respectivos valores de desvio padrão.

\section{RESULTADOS E DISCUSSÃO}

\subsection{Análise Microestrutural}

Como continuação dos estudos realizados previamente sobre os efeitos do tempo e temperatura de encharque nas microestruturas e propriedades mecânicas de aços bifásicos produzidos através de recozimento intercrítico contínuo e direto, apresentados anteriormente por Silva et. al. [15,16], na Figura 3 são exibidas as imagens obtidas por microscopia eletrônica de varredura (MEV) das amostras tratadas por recozimento intercrítico direto a $740^{\circ} \mathrm{C}$ e $780^{\circ} \mathrm{C}$ e na figura 4 são apresentadas as frações volumétricas de cada um dos constituintes para 0 respectivo processamento térmico.

É possível observar o aumento da fração volumétrica do constituinte MA nas duas temperaturas de processamento com o aumento do tempo, de forma mais acentuada para $740^{\circ} \mathrm{C}$ e a diminuição da fração volumétrica da ferrita. Também é possível perceber um aumento nos tamanhos das ilhas de martensita com a elevação do tempo de encharque para as amostras tratadas a $740^{\circ} \mathrm{C}$, o que não é observado nas amostras tratadas a $780^{\circ} \mathrm{C}$. Esse fenômeno também foi observado por Mazaheri et al. em seus estudos [17]. As amostras tratadas a $780^{\circ} \mathrm{C}$ apresentaram maior fração volumétrica de martensita, em todos os tempos de encharque, se comparadas às amostras a $740^{\circ} \mathrm{C}$, de acordo com os resultados verificados por Ghaheri et al. e Xu et al. [9,12] 


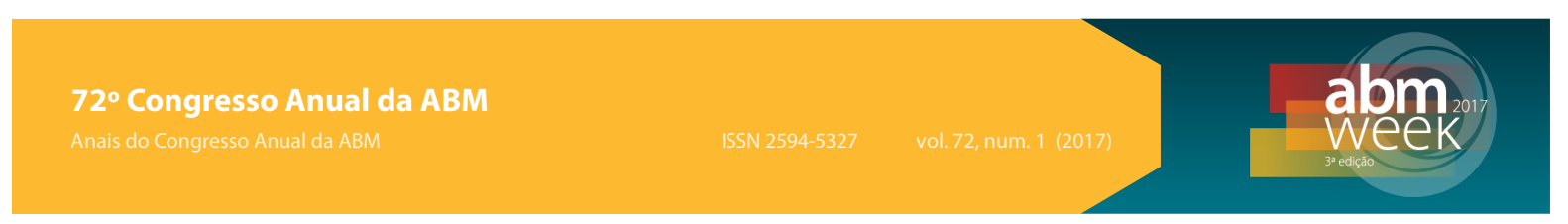

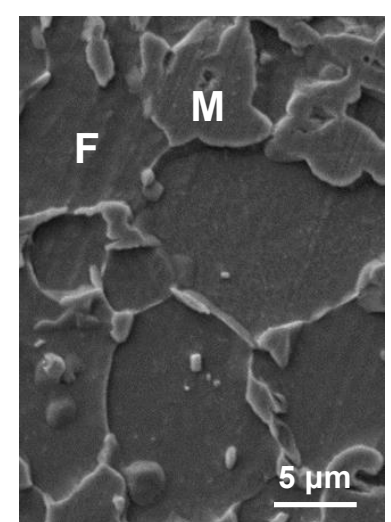

(a)

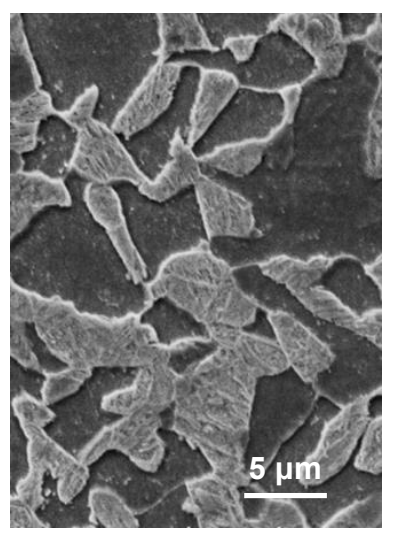

(b)

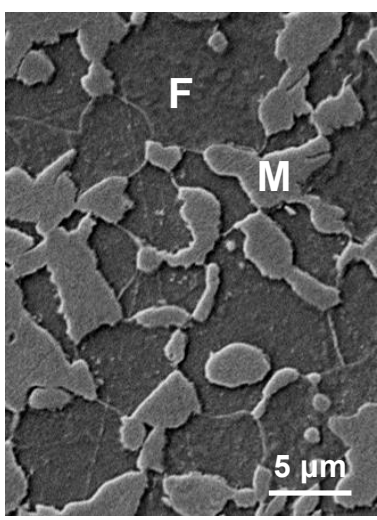

(c)

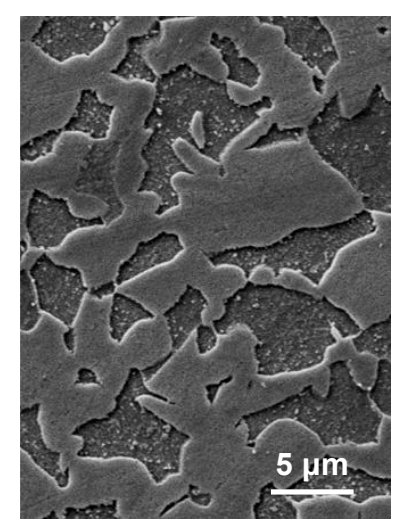

(d)

Figura 3: Imagens de MEV de amostras tratadas por Recozimento Intercrítico Direto.

F - Ferrita; $M$ - Constituinte MA.

(a) $740^{\circ} \mathrm{C}-2 \min$, (b) $740^{\circ} \mathrm{C}-30 \min ,(c) 780^{\circ} \mathrm{C}-2 \min$, (d) $780^{\circ} \mathrm{C}-30 \min$.
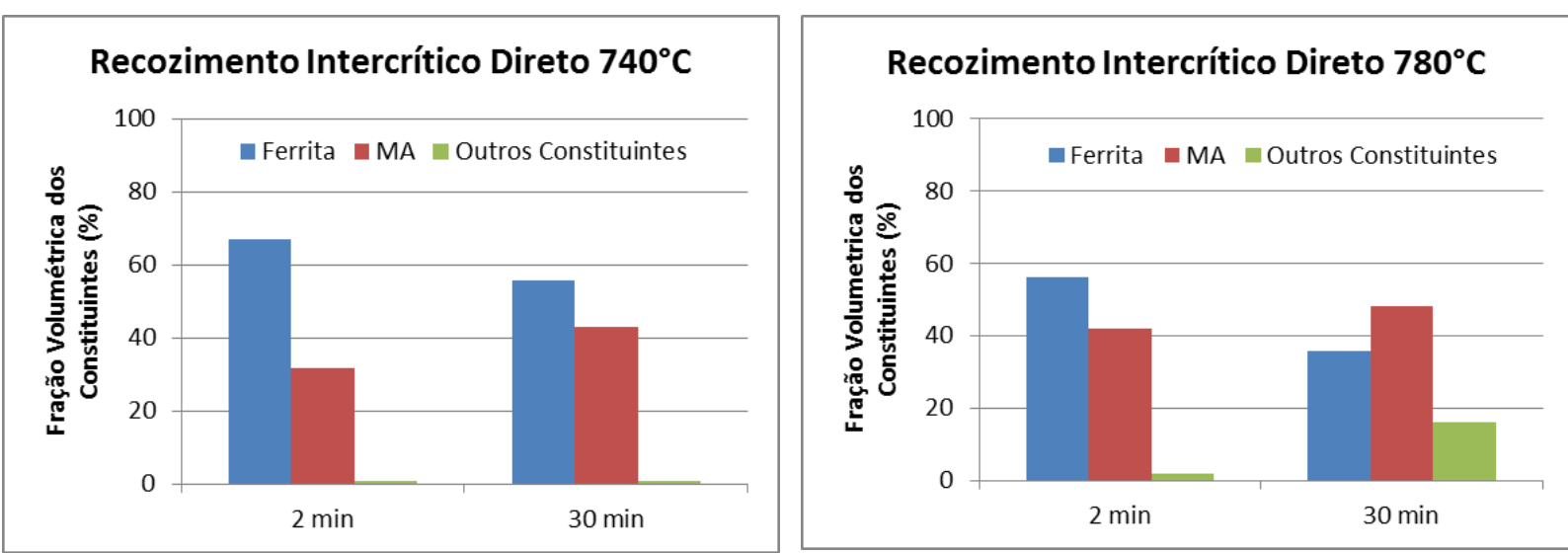

Figura 4: Fração Volumétrica dos Constituintes das amostras submetidas ao Recozimento Intercrítico Direto.

Nas figuras 5 e 6 podem ser observadas as imagens obtidas por MEV das amostras tratadas por recozimento intercrítico contínuo a $740^{\circ} \mathrm{C}$ e $780^{\circ} \mathrm{C}$ e as frações volumétricas dos constituintes das amostras tratadas, respectivamente. É possível verificar que para temperatura de $740^{\circ} \mathrm{C}$ o aumento do tempo de encharque provocou elevação na fração volumétrica do constituinte MA e diminuição do tamanho de grão ferrítico, à $780^{\circ} \mathrm{C}$ foi observado comportamento similar ao de $740^{\circ} \mathrm{C}$. A quantidade de constituinte MA gerada no tratamento intercrítico contínuo foi superior àquela desenvolvida no processamento direto. Também é possível observar que quanto maior a temperatura empregada, em ambos os processamentos, menor foi a influência do tempo. Resultados semelhantes aos do presente trabalho foram encontrados por Zhuang et al. [18].

\subsection{Ultramicrodureza nos Constituintes}

Na Figura 7 são mostradas, como exemplos, curvas típicas obtidas no ensaio de ultramicrodureza, por meio do qual o equipamento faz medições de carga por profundidade de penetração. Mesmo todos os ensaios sendo realizados com a mesma carga máxima, previamente determinada, é possível observar variações nas profundidades de penetração nas diferentes áreas analisadas. Na figura 8 são exibidos os valores médios de dureza obtidos nos ensaios com seus respectivos 
desvios padrão para as amostras submetidas ao recozimento intercrítico direto a $740^{\circ} \mathrm{C}$ e $780^{\circ} \mathrm{C}$.

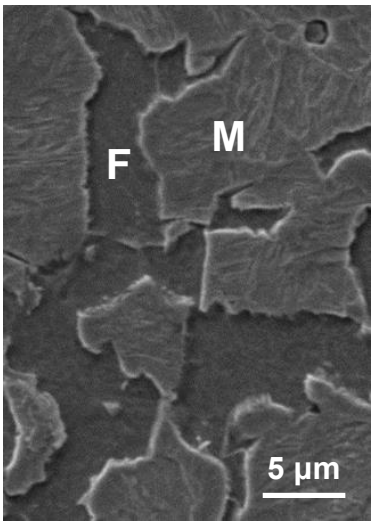

(a)

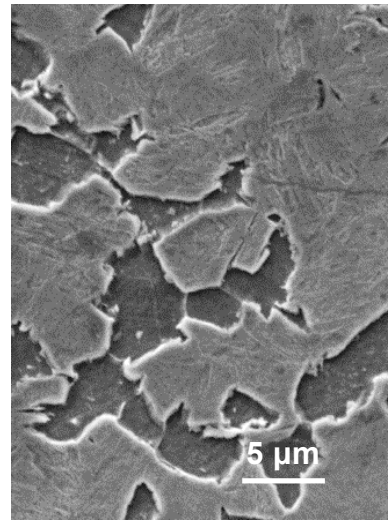

(b)

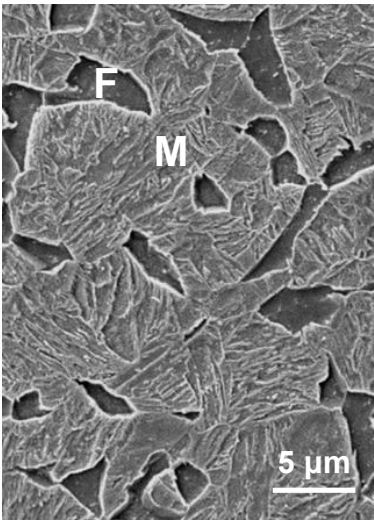

(c)

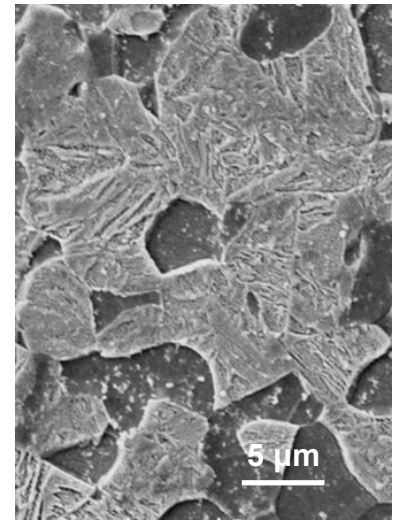

(d)

Figura 5: Imagens de Mev de amostras tratadas por Recozimento Intercrítico Contínuo.

$\mathrm{F}$ - Ferrita; $\mathrm{M}$ - Constituinte MA.

(a) $740^{\circ} \mathrm{C}-2 \min$, (b) $740^{\circ} \mathrm{C}-30 \min$, (c) $780^{\circ} \mathrm{C}-2 \min$, (d) $780^{\circ} \mathrm{C}-30 \min$.

Recozimento Intercrítico Contínuo $740^{\circ} \mathrm{C}$

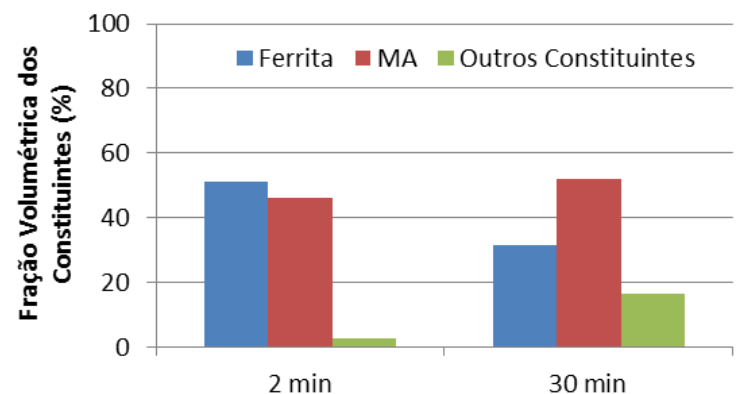

\section{Recozimento Intercrítico Contínuo} $780^{\circ} \mathrm{C}$

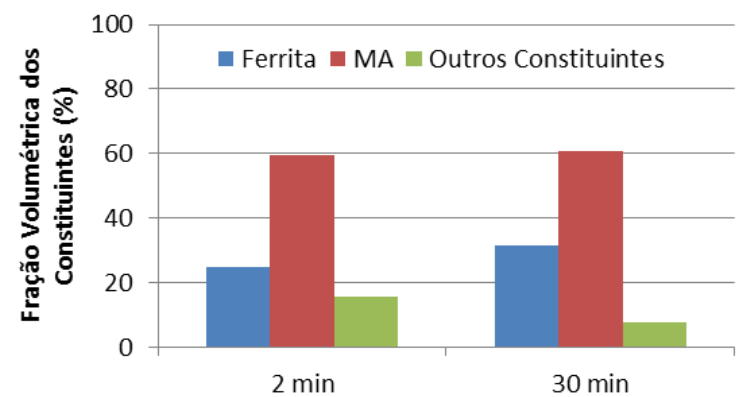

Figura 6: Fração Volumétrica dos Constituintes das amostras submetidas ao Recozimento Intercrítico Contínuo.

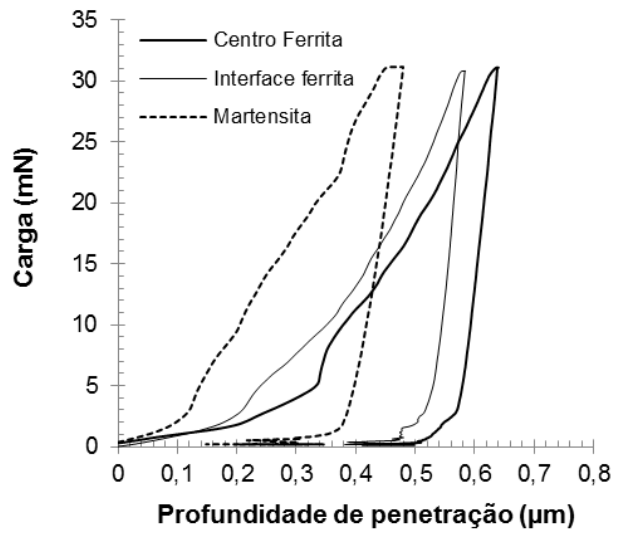

(a)

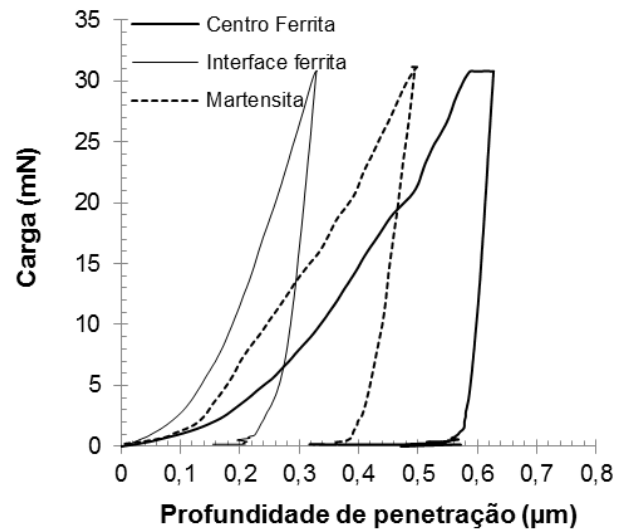

(b)

Figura 7: Curva carga/descarga por penetração instrumentada de amostras submetidas ao Recozimento Intercrítico Direto a $740^{\circ} \mathrm{C}$.

(a) $2 \mathrm{~min}$, (b) $30 \mathrm{~min}$

É possível observar a diferença nos valores de dureza nos constituintes do material. Como esperado, a ferrita apresentou valores inferiores ao constituinte MA. Foi possível observar também que os valores obtidos na interface da ferrita foram 
maiores que os valores obtidos no centro das mesmas, em algumas situações até maiores que do constituinte MA. Esse aumento nos valores na região da interface pode ser explicado pelo aumento da concentração de discordâncias nessa região, devido à expansão volumétrica da martensita durante a sua formação, além do fato de que o material nessa região não é circundado apenas pela fase ferrita, com a presença de constituintes mais duros em seu entorno, dificultando a deformação no local. Resultados similares foram encontrados por Kadkhodapour et al. e GhassemiArmaki et al., trabalhos nos quais foi observado o endurecimento local da ferrita durante a transformação da austenita para martensita $[19,20]$.
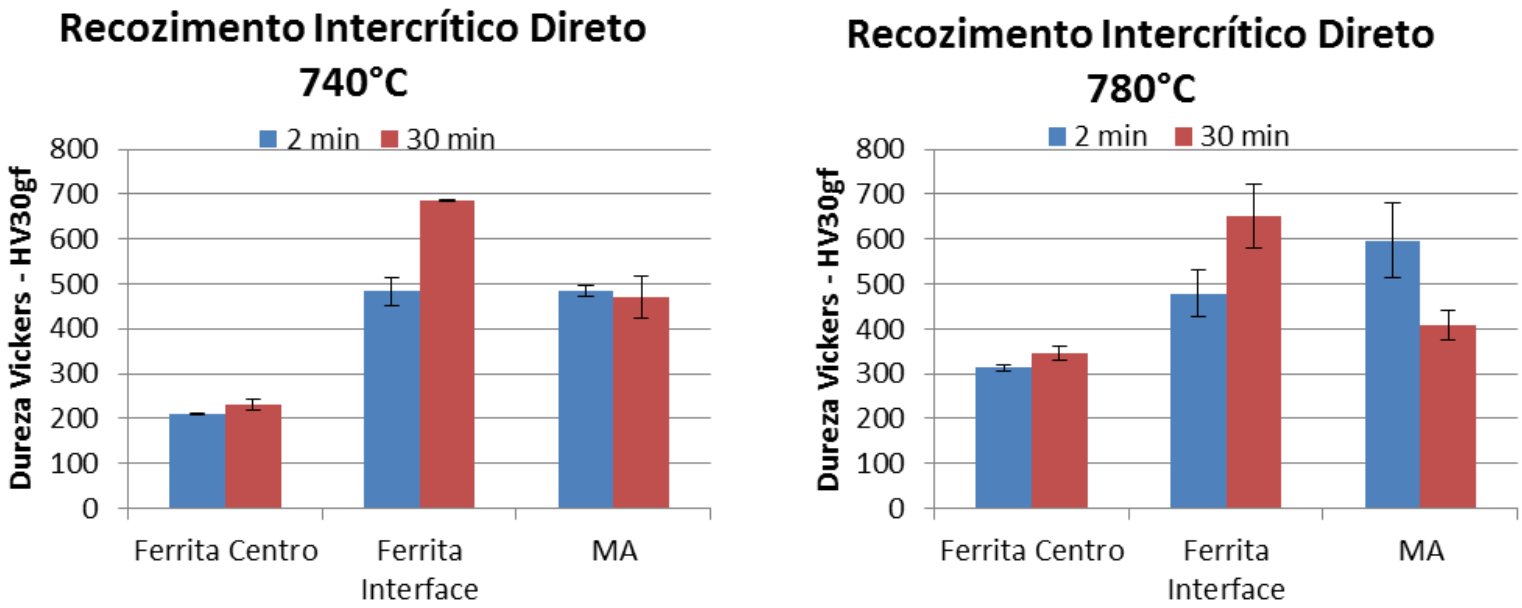

Figura 8: Valores de Dureza Vickers dos constituintes das amostras submetidas ao Recozimento Intercrítico Direto.

Constatou-se também um aumento nos valores de dureza na ferrita e diminuição desses valores no constituinte MA, com o aumento do tempo de encharque no recozimento intercrítico direto. A diminuição nos valores do constituinte MA está relacionado ao aumento do volume da austenita e consequentemente do constituinte MA após o resfriamento, uma vez que esse que irá apresentar um menor teor de carbono, assim apresentando menor valor de dureza. Já a diminuição da fração volumétrica da ferrita faz com que a mesma seja circundada por um maior volume de martensita, aumentando assim o teor de discordâncias presentes na ferrita, em consequência o aumento do seu valor de dureza médio. Movahed et al. observaram em seus estudos que nem sempre o aumento na proporção de martensita resultaria em um aumento nos valores de dureza do material, pois a elevada quantidade martensita, faria com que a mesma apresentasse baixos teores de carbono, e consequentemente menores valores de dureza. [13]

$\mathrm{Na}$ Figura 9 são apresentados os valores médios de dureza dos constituintes das amostras que foram submetidas ao recozimento intercrítico contínuo a $740^{\circ} \mathrm{C}$ e $780^{\circ} \mathrm{C}$ e seus respectivos desvios padrão.

Como no processo anteriormente citado, o centro da ferrita apresentou elevação nos valores de dureza com os respectivos aumentos no tempo e temperatura de encharque. Já a interface da ferrita sofreu alterações apenas com o aumento do tempo de encharque, o que não foi possível verificar com o aumento da temperatura no tempo de $30 \mathrm{~min}$, visto que as ilhas de martensita não apresentam alterações intensas em termos de proporção e morfologia, assim, não influenciando de forma a alterar os valores de dureza na região da interface. Ebrahimian e Banadkouki ao estudarem diferentes regiões de um aço bifásico, observaram variações nos valores 
de dureza ao longo dos grãos de ferrita, aumentando o valor com a aproximação da região com a presença do constituinte MA. [21]

Os valores obtidos do constituinte MA não sofreram grandes alterações devido ao desvio padrão elevado obtido, podendo existir superposição de resultados, o que dificulta a avaliação da microestrutura por meio do método de ultramicrodureza, fenômeno também foi observado por Kadkhodapour et al. [19].
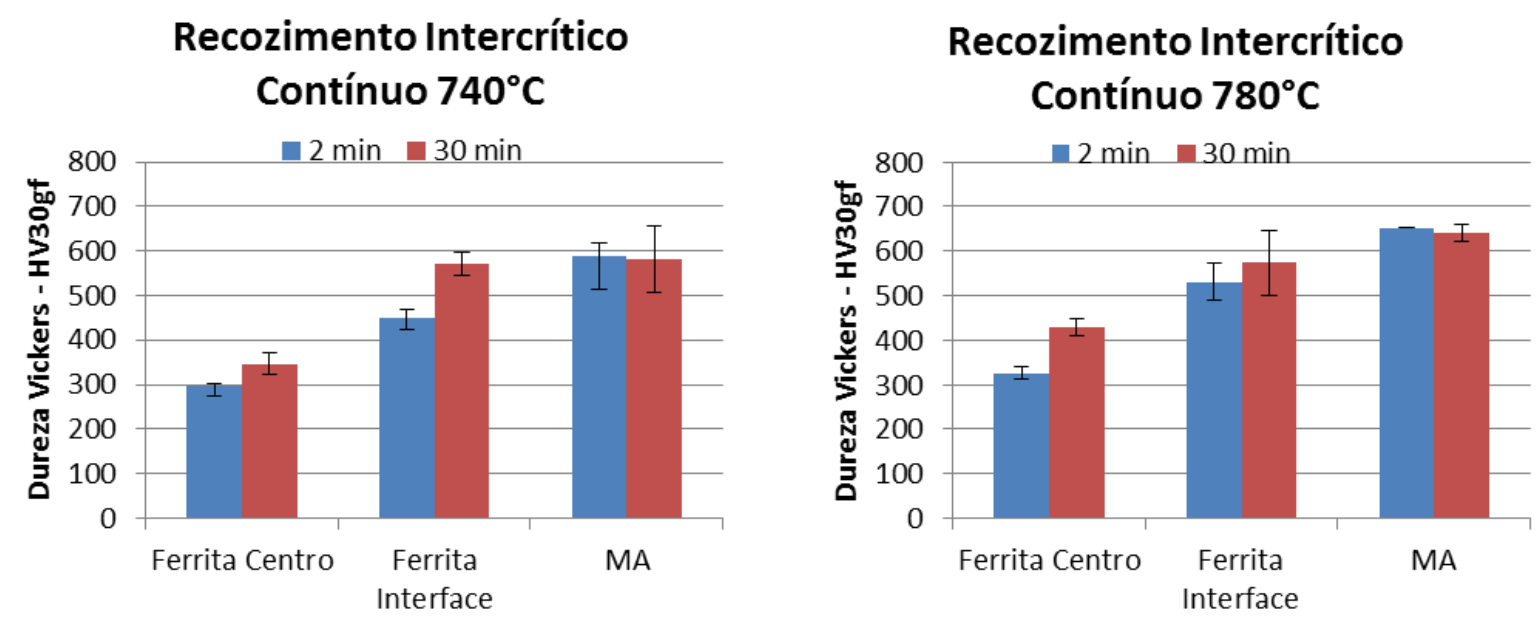

Figura 9: Valores de Dureza Vickers dos constituintes das amostras submetidas ao Recozimento Intercrítico Contínuo.

\section{CONCLUSÃO}

Em conclusão quanto a análise microestrutural para o recozimento intercrítico direto que um maior tempo ou temperatura de encharque maior foi o volume de martensita obtido. Os valores de dureza aumentaram com o aumento do tempo e temperatura de encharque para a ferrita e diminuição para o constituinte MA.

No recozimento intercrítico contínuo o aumento da temperatura promoveu um aumento no volume de martensita. Quanto o tempo de encharque foi significativo apenas para a temperatura de $740^{\circ} \mathrm{C}$, com menor efeito ainda no recozimento intercrítico contínuo. Em geral, o tratamento contínuo apresentou maiores teores de constituinte MA que o tratamento direto. Com valores de dureza neste tratamento foi possível concluir que um aumento nos valores de dureza da ferrita, enquanto a dureza da martensita não foi alterada.

\section{Agradecimentos}

Ao CNPq e à CAPES pela concessão de bolsas de iniciação científica e de Mestrado, à FINEP pelo auxílio financeiro por meio da compra de equipamentos e à MetalTemper pela realização dos tratamentos térmicos.

\section{REFERÊNCIAS}

1 MATLOCK, D. K.; SPEER, J.G.; MOOR, E.D; GIBBS, P.J. Recent developments in advanced high strength steel for automotive applications: an overview. Jestech, v.15, n.1, p. 112, 2012.

2 DIEGO-CALDERON, I.; SANTOFIMIA, M. J.; MOLINA-ALDAREGUIA, J. M.; MONCLUS, M. A.; SABIROV, I. Deformation behavior of a high strength multiphase 
steel at macro- and micro-scales. Materials Science and Engineering A, v. 611, p. 201-211, 2014

3 FONSTEIN, N. Advanced high strength sheet steels: physical metallurgy, design, processing and properties. 1. ed. Switzerland: Springer, 2015. 415p.

4 HOVART, C.D. Advanced steels for lightweight for automotive structures. In: MALLICK, P.K. Materials, design and manufacturing for lightweigth vehicles. New York: Elsevier, 2010.

5 ZHANG, F; RUIMI, A; WO, P. C.; FIELD, D. P. Morphology and distribution of martensite in dual phase (DP980) steel and its relation to the multiscale mechanical behavior. Materials Science and Engineering A, v. 659, p. $93-103,2016$.

6 WANG, W.; LI, M.; HE, C.;WEI, X.;WANG, D.;DU, H. Experimental study on high strain rate behavior of high strength 600-1000 Mpa dual phase steels and $1200 \mathrm{MPa}$ fully martensitic steels. Materials and Design, v. 47, 2013, p. 510-521

7 MAZAHERI, Y; KERMANPUR, A; NAJAFIZADEH, A. A novel route for development of ultrahigh strength dual phase steels. Materials Science and Engineering A, v. 619, p. 1-11, Sep. 2014a.

8 MALEQUE, M. A.; POON, Y. M.; MASJUKI, H.H. The effect of intercritical heat treatment on the mechanical properties of AISI 3115 steel. Journal of Materials Processing Technology, v. 153-154, p. 482-487, 2004.

9 XU, X.; ZWAAG, S.; XU, W. The effect of martensite volume fraction on the scratch and abrasion resistance of aferrite-martensite dual phase steel. Wear, v. 348-349, p. 80 88, 2016.

10 DIAS, A. N. O. Avaliação das propriedades mecânicas de um aço bifásico com diferentes microestruturas. 2013. 94f. Dissertação (mestrado) - Programa de PósGraduação em Engenharia de Materiais, Universidade Federal de Itajubá, Itajubá.

11 TASCA, M. R. B.; MACHADO, M. L. P. Simulação da laminação a quente para a produção de aço bifásico. In: Workshop de Ciência e Tecnologia em Engenharia Metalúrgica e de Materiais do PROPEMM, 2. Agosto, 2012, Vitória. Anais... Vitória: PROPEMM, 2012.

12 GHAHERI, A.; SHAFYEI, A.; HONARMAND, M. Effects of inter-critical temperatures on martensity morphology, volume fraction and mechanical properties of dual phase steels obtained from direct and continuous annealing cycles. Materials and Design, v. 62, p. $305-319$, may 2014.

13 MOVAHED, P. KOLAHGAR, S.; MARASHI, S.P.H.; POURANVARI, M.; PARVIN, N. The effect of intercritical heat treatment temperature on the tensile properties and work hardening behavior of ferrite-martensite dual phase steel sheets. Materials Science and Engineering A, v. 518, p. 1-6, May 2009.

14 COLPAERT, Hubertus. Metalografia dos produtos siderúrgicos comuns. 4. ed. São Paulo: Edgard Blucher, 2008. 652p.

15 SILVA, N.A. N.; LOURENÇO, P. P. C.; MACHADO, E. D. P.; SANTOS, P. S.; AGUILAR, M. T. P.; LOPES, W.; CORRÊA, E. C. S. Efeito do tempo de encharque nos recozimentos intercríticos direto e contínuo na microestrutura e nas propriedades dos aços bifásicos. In: Congresso Brasileiro de Ciência e Engenharia de Materiais, Novembro, 2016, Natal. Anais... Natal: CBECiMat, 2016.

16 SILVA, N.A. N.; LOURENÇO, P. P. C.; SANTOS, P. S.; RESENDE, D. C. S; BRAGA, C.; LOPES, W.; CORRÊA, E. C. S. Influência do tempo e da temperatura de recozimento intercrítico direto no desenvolvimento da microestrutura bifásica de um aço 0,12\%C-0,50\%SI-1,58\%MN. In: Congresso Brasileiro de Engenharia de Fabricação, 2017, Joinville. Anais... Joinville: COBEF, 2017.

17 MAZAHERI, Y; KERMANPUR, A; NAJAFIZADEH, A. Nanoindentation study of ferritemartensite dual phase steels developed by a new thermomechanical processing.

Materials Science and Engineering A, v. 639, p. 8-14, May 2015.

18 ZHUANG, L.; WU, D.; WEI, L.; HUANHUAN, Y.; ZHENYAO, S.; LEI, L. Effect of holding time on the microstructure and mechanical properties of dual-phase steel during 
intercritical annealing. Journal of Wuhan University of Technology-Mater. Sci., v. 30 , n. 1, p. $156-161,2015$.

19 KADKHODAPOUR, J.; SCHMAUDER, S. RAABE, D.; ZIAEI-RAD, S.; WEBER, U.; CALCAGNOTTO, M. Experimental and numerical study on geometrically necessary dislocations and non-homogeneous mechanical properties of the ferrite phase in dual phase steels. Acta Materialia, v. 59, p. 4387 - 4394, 2011.

20 GHASSEMI-ARMAKI, $\mathrm{H}$. et al. Deformation response of ferrite and martensite in a dualphase steel. Acta Materialia, v. 62, p. 197-211, 2014.

21 EBRAHUMIAN, A., GHASEMI BANADKOUKI, S. S. Effect of alloying element partitioning on ferrite hardening in a low alloy ferrite-martensite dual phase steel. Materials Science and Engineering A, v. 677, p. 281 - 289, 2016. 